\title{
Nível de atividade física de portado- res de diabetes mellitus tipo 2 (DM2) em comunidade carente no Brasil
}

\author{
Level of physical activity of carriers of diabetes mellitus tipo 2(DM2) \\ in a poor community in Brazil
}

Dênis Marcelo Modeneze ${ }^{1}$, Roberto Vilarta ${ }^{2}$, Érika da Silva Maciel ${ }^{3}$, Jaqueline Girnos Sonati ${ }^{4}$, Marcos Eduardo Sales Nunes de Souza ${ }^{5}$, Estela Marina Alves Boccaletto 6

\begin{abstract}
RESUMO
Modelo do estudo: Estudo transversal observacional. Objetivo: O objetivo deste trabalho foi identificar o nível de atividade física de indivíduos portadores de diabetes mellitus, pertencentes às comunidades carentes. Métodos: Foram entrevistados 101 indivíduos portadores de diabetes tipo 2, de ambos os sexos com média de idade de 63,6 anos $( \pm 11,4)$, participantes de uma associação municipal de diabéticos, no período de maio a junho de 2009. As variáveis coletas foram nível de atividade física, com a aplicação do Questionário Internacional de Atividade Física forma curta (IPAQ), variáveis demográficas, socioeconômicas e de saúde com aplicação de questionário estruturado. Resultados: Observouse que a maioria era do sexo feminino, casada, aposentada, baixo nível socioeconômico e pouca escolaridade. $87,3 \%$ referiram pelo menos uma comorbidade, prevalecendo hipertensão arterial (64\%), problemas na coluna (38\%) e artrose (31\%). Quanto à prática de atividade física, o grupo estudado apresentou elevados níveis (21,8\% Muito Ativos e 65,3\% Ativos). Conclusão: Os sujeitos do estudo apresentaram elevados níveis de atividade física onde os homens relataram participação significativamente maior na prática da caminhada do que as mulheres, relação que se inverte nas atividades moderadas e vigorosas onde as mulheres se destacaram. Porém o bom escore detectado pelo instrumento não minimiza a importância de se desenvolver programas com atividades físicas orientadas e específicas a esta população.
\end{abstract}

Palavras-chave: Diabetes Mellitus Tipo 2. Planos e Programas de Saúde. Atividade Física.

1. Doutor em Educação Física pela Universidade Estadual de Campinas (UNICAMP) Campinas, SP.Faculdade de Educação Física.

2. Professor Titular. Departamento de Estudos da Atividade Física Adaptada, Faculdade de Educação Física. UNICAMP.

3. Professora Doutora do curso de Educação Física CEULP/ ULBRA, Palmas, TO. Doutora em Ciências. Centro de Energia Nuclear na Agricultura. Universidade de São Paulo (USP), Departamento de Tecnologia de Alimentos da Escola Superior de Agricultura Luiz de Queiroz (ESALQ)."

4. Mestre em Educação Física, UNICAMP. Faculdade de Educação Física.

5. Mestre em Educação Física, UNICAMP. Faculdade de Educação Física.

6. Doutora em Educação Física, UNICAMP. Faculdade de Educação Física.
Correspondência:

Prof. Dr. Dênis M. Modeneze UNICAMP - Campus "Zeferino Vaz", Faculdade de Educação Física, Departamento de Estudos da Atividade Física Adaptada, Rua Érico Veríssimo, 701, Caixa Postal 6134, 13083-851 - Campinas, SP, Brasil. e-mail: modeneze@uol.com.br

Artigo recebido em 17/10/2011 Aprovado para publicação em 27/03/2012 


\section{Introdução}

O estilo de vida contemporâneo tem acarretado problemas de saúde na população em geral, com frequente ocorrência de doenças crônicas não transmissíveis -DCNT em várias faixas etárias.

Nesse sentido, destaque tem sido dado à diabetes mellitus tipo 2 (DM2) que, nos países em desenvolvimento, apresenta tendência de aumento da prevalência, cujo reflexo negativo sobre a qualidade de vida e o impacto da doença aos sistemas de saúde são imensuráveis. ${ }^{1}$

A Organização Mundial de Saúde (OMS) estima em 143 milhões o número de portadores de DM2 no mundo. A projeção para o ano 2025 é de que a patologia atinja trezentos milhões de pessoas, em grande parte nos países em desenvolvimento, principalmente devido ao crescimento e envelhecimento da população, à obesidade, aos fatores dietéticos e ao sedentarismo. No Brasil, segundo estimativas do Ministério da Saúde, existem aproximadamente cinco milhões de portadores de DM2, $90 \%$ dos quais do tipo 2 e 5 a $10 \%$ do tipo $1 .^{2}$

Envelhecimento populacional, consumo alimentar inadequado, adoção de estilo de vida sedentário e aumento do sobrepeso e da obesidade, têm sido apontados como possíveis fatores associados com as elevadas taxas de DM2 observadas no Brasil, ressaltando a relevância da implementação e adequação de programas de prevenção primária em serviços públicos de saúde. ${ }^{1}$

O DM2 caracterizado pelo desequilíbrio no metabolismo de carboidratos, lipídeos e proteínas, possui um tratamento específico e complexo que envolve a manutenção de uma dieta adequada, a prática sistemática de exercícios físicos, o uso de agentes hipoglicêmicos e/ou insulina quando necessário a fim de auxiliar no constante controle da glicemia, o que requer uma mudança no estilo de vida individual. ${ }^{3}$

A importância da atividade física regular e do exercício sistematizado para a prevenção de doenças e promoção da saúde está definitivamente aceita ${ }^{4,5}$, sendo que essa prática orientada tem sido indicada em conjunto com a dieta e a medicação para tratamento da diabete, auxiliando na motivação e na mudança de hábitos e comportamentos. ${ }^{6}$

Outras evidências sugerem que o sedentarismo, potencializado pelo estilo de vida contemporâneo, seja um fator de risco tão importante quanto a dieta inadequada na etiologia da obesidade, estabelecendo uma relação direta e positiva com o aumento da incidência do DM2 em adultos, independentemente do índice de massa corporal ou do histórico familiar. Dados recentes demonstram que o controle de peso corporal e o aumento da prática da atividade física diminuem a resistência à insulina, e por consequência diminuem as chances de desenvolvimento da patologia e o acúmulo de comorbidades. ${ }^{7}$

Vários estudos apontam uma representativa relação dos efeitos da atividade física e do exercício físico para o bem-estar das pessoas, quer sejam crianças, adultos ou idosos, portadores ou não de patologias. ${ }^{8-14}$

Deste modo, no momento em que programas de promoção da atividade física para grupos populacionais começam a ganhar notoriedade em nosso país e considerando que as pesquisas que abordam esta temática são raras e/ou discutíveis, tivemos por objetivo neste estudo observacional com o grupo de adultos cadastrados na Associação de Diabéticos da cidade de Santa Bárbara d'Oeste-SP/Brasil, avaliar o nível de atividade física e verificar relações dessa variável dependente com os aspectos demográficos, socioeconômicos e com as condições de saúde dessa população, a fim de colaborar com o conhecimento dirigido a prevenção e controle do DM2.

\section{Metodologia}

Foi realizado um estudo de corte transversal exploratório em uma população de 101 portadores de DM2 de ambos os sexos, cadastrados na Associação de Diabéticos da cidade de Santa Bárbara d'Oeste, São Paulo, Brasil. Os critérios de inclusão foram: responder espontaneamente os questionários, mediante assinatura de consentimento livre e esclarecido e não estar tomando nenhuma medicação que comprometesse o preenchimento dos questionários. Foram desconsiderados os dados dos sujeitos incapazes de responderem às perguntas, e os que por qualquer motivo abandonaram a pesquisa.

Para a coleta de dados foram utilizados protocolos auto-aplicados sobre informações de identificação e socioeconômicas constando sexo, idade, estado civil, situação ocupacional, escolaridade e nível socioeconômico conforme os critérios de classificação econômica do Brasil. ${ }^{15}$

Para a avaliação dos níveis gerais de atividade física foi aplicado o Questionário Internacional de Atividade Física (IPAQ), versão 8, forma curta e sema- 
na normal, desenvolvido pela OMS, validado, avaliado e utilizado no Brasil e em outros países ${ }^{16,17}$, contendo 8 perguntas em relação à frequência e duração da realização de atividades físicas moderadas, vigorosas e de caminhada tendo como referência a última semana. O nível de atividade física foi classificado segundo o consenso realizado entre o CELAFISCS e o Center for Disease Control (CDC) que considera os critérios de frequência e duração das atividades físicas realizadas, classificando as pessoas em cinco categorias: Muito Ativo, Ativo, Insuficientemente Ativo A, Insuficientemente Ativo B e Sedentário. ${ }^{17}$

Após explanação sobre o projeto de pesquisa, os portadores de DM2 preencheram os questionários e, para aumentar a confiabilidade das respostas, os dados foram conferidos, um a um, pelos examinadores em conjunto com cada indivíduo. Os examinadores foram submetidos a um treinamento antes de conferirem se todos os itens dos questionários foram preenchidos.

O tratamento estatístico foi composto por análise descritiva baseada em medidas de tendência central, dispersão e por distribuição de frequências. Os dados foram analisados no programa estatístico SPSS versão 10.0 .

Esta pesquisa foi aprovada pelo Comitê de Ética em Pesquisa da Faculdade de Ciência Médicas da Unicamp (455/2008) e a todos os grupos participantes foi oferecida uma devolutiva dos resultados com palestra gratuita sobre temas relacionados à saúde do portador de DM2.

\section{Resultados}

As características sociodemográficas dos 101 portadores de DM2 frequentadores da ADSBO estão descritas na Tabela 1, tendo sido observada nessa população a maior frequência de sujeitos do sexo feminino $(83,2 \%)$, casados $(45,5 \%), 40,6 \%$ na faixa etária abaixo dos 60 anos e $35,6 \%$ acima de 70 anos (média de $63,6 \pm 11,4$ anos) e aposentados $(63,4 \%)$. Sobre a escolaridade, quase a metade dos sujeitos não possuíam o ensino primário completo (49,5\%). Em relação ao nível socioeconômico a maioria se concentrou nas classes C2 (30,7\%) e D (43,6\%) conforme os critérios de classificação socioeconômicos do Brasil. ${ }^{15}$

Em relação aos dados sobre as condições de saúde, a frequência de doenças auto-referidas, descrita na Figura 1, mostrou que a maioria dos portadores de DM2 $(87,3 \%)$ referiu pelo menos uma comor-

\section{Tabela 1}

Frequências e percentuais de variáveis demográficas e socioeconômicas dos portadores de DM2 participantes da ADSBO, Piracicaba, SP, Brasil.

\begin{tabular}{lll}
\hline Variável & $\mathrm{N}^{\circ}$ & $\%$ \\
\hline $\begin{array}{c}\text { Idade (anos) } \\
<60\end{array}$ & 41 & 40,6 \\
$60-64$ & 06 & 05,9 \\
$65-69$ & 18 & 17,8 \\
$>70$ & 36 & 35,6
\end{tabular}

Sexo

Feminino

83,2

Masculino

17

16,8

Estado Civil

Casado

46

45,5

Viúvo

38

37,6

Solteiro

05

05,0

Separado

07

06,9

Ocupação

$\begin{array}{lll}\text { Aposentado } & 64 & 63,4 \\ \text { Trabalhando } & 18 & 17,8 \\ \text { Pensionista } & 10 & 09,9 \\ \text { Desempregado } & 05 & 05,0 \\ \text { Afastado } & 03 & 03,0\end{array}$

Escolaridade

$\begin{array}{lll}\text { Primário Incompleto } & 50 & 49,5 \\ \text { Primário Completo } & 31 & 30,7 \\ \text { Ginásio Completo } & 16 & 16,8 \\ \text { Colegial Completo } & 11 & 10,9 \\ \text { Superior Completo } & 02 & 02,0\end{array}$

Nível Sócio-Econômico*

\begin{tabular}{lll} 
B2 & 07 & 06,9 \\
C1 & 17 & 16,8 \\
C2 & 31 & 30,7 \\
D & 44 & 43,6 \\
E & 02 & 02,0 \\
\hline
\end{tabular}

* Critério de Classificação Econômica Brasil (ABEP, 2008) ${ }^{15}$. 


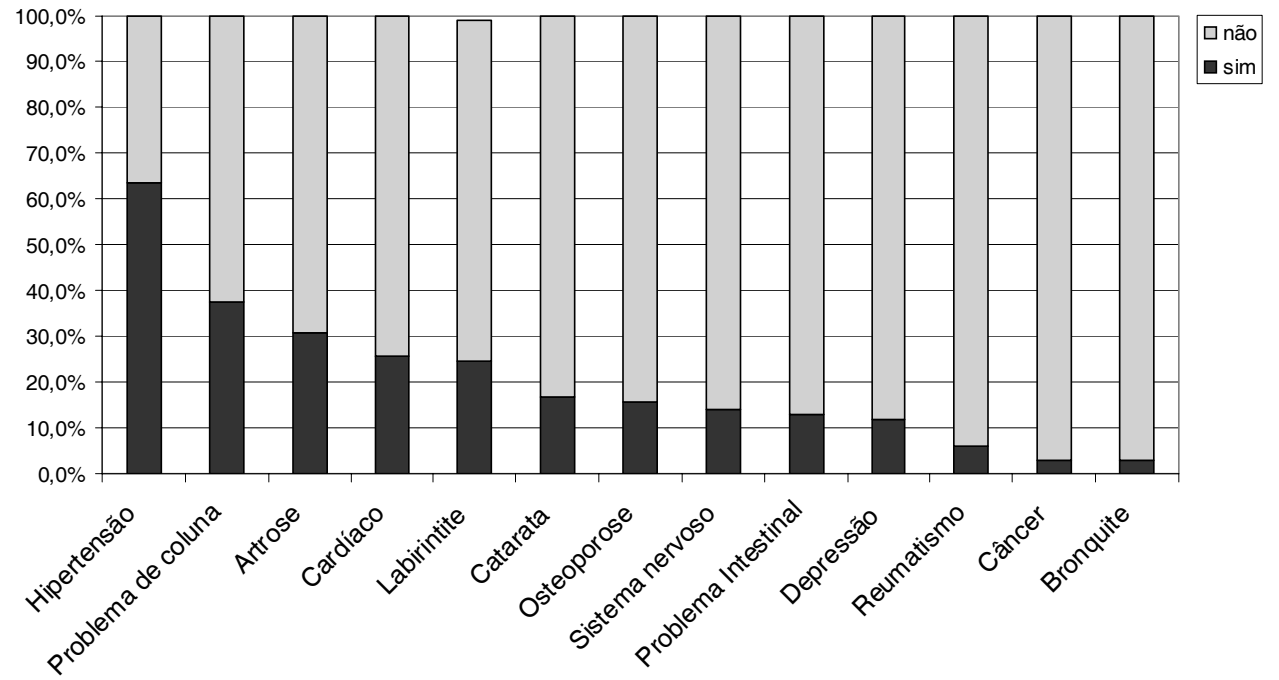

Figura 1: Percentual das doenças auto-referidas pelos 101 portadores de DM2 participantes da ADSBO, Piracicaba, SP, Brasil.

bidade, tendo havido maior prevalência da hipertensão arterial $(64 \%)$, problemas na coluna $(38 \%)$ e artrose $(31 \%)$.

A análise das variáveis relacionadas ao diabetes mostrou que $55,2 \%$ da população referiram ter contraído a doença há pelo menos 10 anos, já o uso de medicamentos para controle foi apontado por $94,1 \%$ da população. Com relação ao comportamento preventivo, $83 \%$ dos sujeitos referiram adotar uma dieta balanceada, mas apenas $20,8 \%$ costumavam verificar a glicemia semanalmente.

Quanto ao nível de atividade física, a maioria da população estudada referiu ser ativa $(65,3 \%)$ conforme critérios de classificação adotados num consenso entre o CELAFISCS e o $\mathrm{CDC}^{17}$, dados que estão expostos em sua totalidade na Tabela 2.

Na Tabela 3 apresentamos a participação percentual da caminhada, das atividades físicas moderadas e das atividades físicas vigorosas totalizadas em minutos por semana.

\section{Discussão}

O presente estudo verificou que a maioria da população era do sexo feminino, casada, aposentada, nível socioeconômico baixo e pouca escolaridade. O fato da elevada presença feminina no grupo avaliado $(83,2 \%)$ pode estar associado não apenas ao processo de feminilização da velhice, visto a maior longevidade e superioridade numérica das mulheres idosas sobre os homens idosos, mas também por serem mais envolvidas social e afetivamente, o que contrasta à

\section{Tabela 2}

Distribuição do Nível de Atividade Física dos portadores de DM2 participantes da ADSBO, SP, Brasil.

\begin{tabular}{lll}
\hline Categoria* & $\mathrm{n}$ & $\%$ \\
\hline Muito Ativo & & \\
Masculino & 04 & 23,5 \\
Feminino & 18 & 21,4 \\
Total & 22 & 21,8
\end{tabular}

Ativo

$\begin{array}{lll}\text { Masculino } & 10 & 58,8 \\ \text { Feminino } & 56 & 66,7 \\ \text { Total } & 66 & 65,3\end{array}$

Insuficientemente Ativo A

$\begin{array}{lll}\text { Masculino } & 1 & 05,9 \\ \text { Feminino } & 5 & 06,0 \\ \text { Total } & 6 & 05,9\end{array}$

Insuficientemente Ativo B

$\begin{array}{lll}\text { Masculino } & 2 & 11,8 \\ \text { Feminino } & 4 & 04,8 \\ \text { Total } & 6 & 05,9\end{array}$

Sedentários

$\begin{array}{lll}\text { Masculino } & 0 & 00,0 \\ \text { Feminino } & 1 & 01,2 \\ \text { Total } & 1 & 01,0\end{array}$

* Critério de Categorização segundo o consenso realizado entre o CELAFISCS e CDC ${ }^{17}$. 


\section{Tabela 3}

Distribuição dos resultados da população nos diferentes tipos de atividade física.

\begin{tabular}{lcc}
\hline Tipo de Atividade & média min/sem & $\%$ \\
\hline Caminhada & 392,57 & 34,4 \\
Atividades Moderadas & 570,53 & 50,2 \\
Atividades Vigorosas & 175,83 & 15,4 \\
Total & 1138,93 & 100,0 \\
\hline
\end{tabular}

média $\mathrm{min} / \mathrm{sem}=$ média das atividades físicas em minutos por semana

forma de ser dos homens idosos que mostram resistência em participar de novas atividades de cunho cultural, educacional e lúdico após a aposentadoria. ${ }^{18-21}$

A média da idade dos entrevistados foi de 63,6 ( \pm 11,4 anos), superior à encontrada em estudo com portadores de DM2 realizado no Rio Grande do Sul, entre 1999 e 2000, no qual a média de idade dos indivíduos participantes foi de 44 anos. ${ }^{22} \mathrm{O}$ aumento da prevalência de diabetes que vem ocorrendo nos países desenvolvidos, principalmente nas faixas etárias mais avançadas, se deve ao aumento da expectativa de vida. Em contrapartida, nos países em desenvolvimento como o Brasil, por exemplo, o crescimento do diabetes é observado em praticamente todas as faixas etárias, mas principalmente no grupo de 45 a 64 anos, com estimativa de ter sua prevalência triplicada nos próximos vinte anos, acarretando um custo maior para os serviços de saúde, além do impacto negativo sobre a qualidade de vida. ${ }^{7}$ Entretanto o estudo realizado por Pelegrini et al ${ }^{23}$ destaca que idosos com excesso de peso referem mais diabetes independentemente da faixa etária, o que minimiza a importância da variável idade como fator de risco para a doença.

Os dados obtidos para escolaridade mostram que a maioria $(49,5 \%)$ foi considerada analfabeta funcional, apresentando ao máximo três anos de estudo. Dados similares foram encontrados em estudo realizado em Ribeirão Preto ${ }^{19}$ com indivíduos portadores de DM2 no ano de 1999, no qual 82,7\% dos entrevistados tinham o primeiro grau incompleto. Outro estudo, realizado no Rio Grande do Sul, encontrou resultados similares onde cerca de $42 \%$ dos indivíduos não completaram o ensino fundamental. ${ }^{22}$ Essa condição, acrescida de outras relacionadas aos aspectos socioeconômicos como renda na aposentadoria, expõem uma situação de fragilidade da pessoa que de- manda atenção à própria saúde, em especial na condição do diabetes, o que requer cuidado especial na proposição de estratégias utilizadas para verificar o quanto está sendo eficaz a comunicação nas orientações da equipe multidisciplinar de saúde. ${ }^{25}$ Estudo realizado por Souza et al. ${ }^{26}$ verificou tendência para o aumento da prevalência do diabetes em sujeitos com baixo grau de escolaridade. A importância de analisar o grau de instrução se deve ao fato de que a condição da escolaridade pode dificultar o acesso às informações e trazer menores oportunidades de aprendizagem relacionadas com a saúde, pois os pacientes portadores de DM2 desenvolvem, em grande parte, o seu próprio cuidado. ${ }^{27}$

A baixa renda é um fator que pode comprometer as condições de saúde dos portadores de DM2. ${ }^{28}$ Em nosso estudo verificamos que a principal fonte de renda da população foi o benefício econômico da aposentadoria $(63,4 \%)$ dado corroborado com outros estudos realizados com populações de baixa renda. ${ }^{28,29}$ Contudo, 94,1\% dos portadores de DM2 faziam uso regular de medicamentos o que, na maioria das vezes, compromete o orçamento doméstico, podendo causar prejuízo tanto no tratamento medicamentoso quanto no dietético. Esta situação é ainda pior para aqueles de classes econômicas inferiores "D" e "E" (45,6\%), que dependem de instituições públicas, associações de apoio a portadores de DM2 e de familiares para aquisição de medicamentos.

Entendemos que, diante das características socioeconômicas observadas, somadas às limitações impostas pela doença, eleva-se a importância das ações de educação em saúde, realizadas em grupos de apoio aos portadores de DM2, foco de nossa pesquisa, principalmente no que se refere às orientações para o autocuidado, respeitando o contexto social e cultural dessa comunidade, aproveitando os recursos disponíveis, de acordo com a própria realidade, para adquirir hábitos mais saudáveis, implementando os cuidados do paciente portador de DM2.

Com relação aos dados sobre as condições de saúde, a maioria dos portadores de DM2 $(87,3 \%)$ referiu pelo menos uma comorbidade, prevalecendo a hipertensão arterial (64\%), seguida por problemas na coluna (38\%) e artrose (31\%). Esses achados corroboram com o estudo descritivo de Lima-Costa et al $^{30}$ sobre as condições de saúde da população brasileira onde, $69 \%$ dos sujeitos, acima de 60 anos, relataram ter pelo menos uma doença crônica, sendo hipertensão e artrite as mais comuns. 
Existe um consenso entre os autores, de que a hipertensão arterial é cerca de duas vezes mais frequente entre indivíduos portadores de DM2, quando comparados à população em geral, e está presente em 50\% dos pacientes no momento do diagnóstico da DM2. ${ }^{31-34}$ Estudos demonstraram que a hipertensão arterial associada à DM2 multiplica o risco de morte cardíaca $^{35}$, portanto seu controle eficiente reduz, de forma significativa, os acidentes encefálicos, os óbitos relacionados à diabete, insuficiência cardíaca, complicações microvasculares e perda visual. ${ }^{36}$

Quanto à prática de atividade física, o grupo de portadores de DM2 desse estudo apresentou elevados níveis (21,8\% Muito Ativos e 65,3\% Ativos), dados que corroboram com os da pesquisa realizada com mulheres portadoras de doença vascular periférica que encontrou uma prevalência de $74,7 \%$ de ativas fisicamente. ${ }^{37}$ Várias pesquisas ${ }^{38,39,40}$ ressaltam a importância da prática regular de atividade física na melhora da aptidão física, por requerer um nível mínimo de força muscular, flexibilidade, coordenação e equilíbrio, refletindo em resultados positivos para a manutenção da capacidade funcional.

No desmembramento do IPAQ observamos que na prática da caminhada os homens relataram uma participação maior, com média de 833,82 minutos por semana $( \pm 915,94 \mathrm{~min} / \mathrm{sem})$, do que as mulheres cuja média foi de 303,27 minutos por semana ( \pm 393,18 $\mathrm{min} / \mathrm{sem}$ ). Já nas atividades moderadas e vigorosas foram as mulheres que se destacaram, atingindo médias de 637,01 ( $\pm 857,63 \mathrm{~min} / \mathrm{sem})$ e 200,05 ( $\pm 434,78$ $\mathrm{min} / \mathrm{sem}$ ) respectivamente, contra médias de 242,06 $( \pm 358,17 \mathrm{~min} / \mathrm{sem})$ e $56,18( \pm 103,37)$ dos homens, alcançando diferenças significativas $(\mathrm{P}<0,05)$.

A prática de atividade física diminui com a idade. ${ }^{41}$ Nossos achados confirmam essa posição da Organização Mundial da Saúde e são semelhantes aos do estudo realizado por Hallal et al. ${ }^{42}$, onde a inatividade física esteve positivamente associada com a idade. Encontramos uma correlação negativa $(-0,235)$ entre as variáveis idade e prática de atividades vigorosas onde, quanto menor a faixa etária da população de portadores de DM2, maior era a quantidade de atividades vigorosas praticadas na semana $(\mathrm{P}<0,05)$, dados que podem ser justificados pelo fenômeno do envelhecimento e complicações do diabetes no decorrer da vida.

A caminhada parece estar entre as atividades físicas mais praticadas, condição confirmada pelo trabalho de Caspersen, Kriska e Dearwater ${ }^{43}$ que compilaram informações de estudos epidemiológicos rea- lizados na Inglaterra, Estados Unidos e Holanda. Esses autores constataram a prática da caminhada variando de $38 \%$ a $72 \%$ nas amostras, aspecto esse confirmado por nosso estudo em $97 \%$ da amostra, representando cerca de $34,4 \%$ do somatório de todas as atividades físicas do IPAQ e com uma média de 392,57 minutos por semana ( $\mathrm{min} / \mathrm{sem})$.

Observamos também que na prática de atividades moderadas os portadores de DM2, classificados abaixo dos 60 anos, relataram uma participação maior, com média de 865,79 minutos por semana $( \pm 583,08 \mathrm{~min} / \mathrm{sem})$, do que os de 60 anos ou mais velhos cuja média foi de 465,75 minutos por semana ( $\pm 755,78 \mathrm{~min} / \mathrm{sem})$. Também nas atividades vigorosas as médias atingidas nas idades menores que 60 anos foram de 287,41 ( $\pm 583,08 \mathrm{~min} / \mathrm{sem})$ contra médias de 99,58 ( $\pm 169,48 \mathrm{~min} / \mathrm{sem})$ para os maiores ou igual a 60 anos, alcançando também diferenças significativas $(\mathrm{P}<0,05)$.

A intensidade da prática de atividades físicas também é um fator influenciador da aptidão física. Brach et al. ${ }^{44}$ confirmaram essa influência ao apontarem para o fato de que indivíduos que praticavam atividades em intensidades maiores possuíam melhor função física do que aqueles que realizavam atividades de menores intensidades. Já Young, Masaki e Curb $^{45}$ relataram essa mesma influência em seus achados, onde os indivíduos que obtinham um maior gasto de energia durante as atividades físicas sistematizadas apresentavam, com maior frequência, uma melhor aptidão física. Em nosso estudo as atividades mais intensas ou vigorosas não foram muito frequentes na rotina dos portadores de DM2 que realizavam, em média, 175,83 $\mathrm{min} / \mathrm{sem}$, representando apenas $15,4 \%$ do total de atividades físicas praticadas na semana, fato que não descaracteriza o bom nível de atividade física da população estudada.

Com base nos dados desse estudo identificamos o perfil socioeconômico, educacional e de atividade física dos portadores de DM2 e discutimos essas variáveis com os achados da literatura, contudo vale ressaltar que o número de pesquisas no Brasil, utilizando os instrumentos e as classificações empregados no presente estudo, ainda é reduzido, o que dificulta uma discussão mais especifica e ampliada.

\section{Conclusão}

Os sujeitos do estudo apresentaram elevados níveis de atividade física onde os homens relataram participação significativamente maior na prática da 
caminhada do que as mulheres, relação que se inverte nas atividades moderadas e vigorosas onde as mulheres se destacaram. Porém o bom escore detectado pelo instrumento não minimiza a importância de se desenvolver programas com atividades físicas específicas direcionadas a esta população.

Ressaltamos portanto, a importância de uma ação sinérgica entre os setores público, privado e comunidade organizada quando da utilização de informações diagnósticas, como as obtidas por esse estudo, no delineamento, implementação e monitoramento de ações promotoras da saúde.

Grupos de apoio social podem ser importantes parceiros das organizações públicas no desenvolvimento de estratégias de promoção da saúde de forma a ampliar o número de doentes atendidos, porém o levantamento do perfil dos participantes carece de reconhecimento e valorização no desenvolvimento e implementação de programas de saúde populacional.

Assim, na elaboração de atividades voltadas à promoção da saúde em grupos de portadores de DM2 com perfis socioeconômicos e epidemiológicos seme- lhantes aos observados nesse estudo, entendemos ser apropriado a inclusão de palestras e outras formas de educação formal e informal, com metodologia adequada às necessidades e limitações desse público, focando na melhor adaptação do portador de DM2 à família e a mais informações sobre a patologia, buscando alternativas para aumentar a participação de todos.

Declaramos não haver conflitos de interesse pertinentes.

\section{Colaboradores}

DM Modeneze participou da concepção, da elaboração do projeto de pesquisa, supervisão e orientação do desenvolvimento da pesquisa, coleta e análise dos dados e redação final. ES Maciel participou da concepção, coleta e análise dos dados e redação final. R Vilarta foi revisor crítico, orientador e participou da redação final. JG Sonati, EMA Boccaletto e MESN Souza forma revisores críticos e participaram da redação final e da metodologia.

\begin{abstract}
Study Model: It was done an observational and analytical study with a cross-sectional design. Objective: This study focuses on assessing the level of physical activity and verifying if it is related to socioeconomic aspects and health conditions of Diabetes Mellitus type 2 sufferers in a poor community. Methods: A consecutive sampling was carried out with 101 individuals, both male and female, average age being 63.6 ( \pm 11.4 years), and the majority of whom were female, married, retired, lowly educated, and belonging to a low socioeconomic level. The following variables were collected: level of physical activity, with the application of the International Questionnaire of Physical Activity (IPAQ), demographic variables, socioeconomic aspects and health conditions with application of a structured questionnaire. Results: Adding the practice of physical activity, the studied group presented high levels (21,8\% Very Active and $65,3 \%$ Active).Statistical analyses of independent variables suggest an important correlation between age, habit of verifying glycemia, and education level, being level of physical activity the dependent variable. Conclusion: In a view of the correlations observed, in additional of the limitations imposed by the disease, it is highly important that actions on health education be taken amidst professionally operated support groups to help DM sufferers. These actions should include orientations for self care and frequent physical activities, respecting each community's social and cultural contexts, and local living conditions as well.
\end{abstract}

Keywords: Diabetes Mellitus, Type 2. Health Programs and Plans. Motor Activity.

\section{Referências Bibliográficas}

1. King $\mathrm{H}$, Aubert RE, Herman WH. Global burden of diabetes, 1995 - 2025. Diabetes Care. 1998; 21:1414-31.

2. Lojudice FH, Sogayar MC. Células-tronco no tratamento e cura do diabetes mellitus. Ciênc Saúde Coletiva. [online]. 2008; 13:19-21.
3. Peres DS, Franco LJ, Santos MA. Feelings of women after the diagnosis of type 2 diabetes. Rev Latinoam Enferm. [online]. 2008; 16:101-8.

4. (CDC) U.S. Department of Health and Human Services, Center for Disease Control and Prevention, National Center for Chronic Disease Prevention and Health Promotion Services. Physical activity and health: A report of the Surgeon General. Atlanta, GA: $1996 ; 146-8$. 
5. NHI - National Institutes of Health. NIH Consensus Statement: Physical Activity and Cardiovascular Health. Hyattsville, MD: National Institutes of Health. 1995; 13:1-33.

6. Praet SFE, Van Rooij ESJ, Wijtvliet A, Boonman de Winter LJM, Enneking TH, Kuipers H, Stehouwer CDA, Van Loon LJC. Brisk walking compared with an individualised medical fitness programme for patients with type 2 diabetes: a randomised controlled trial. Diabetologia. 2008; 51:736-46.

7. Sartorelli DS, Franco LJ. Tendências do diabetes mellitus no Brasil:o papel da transição nutricional. Cad Saúde Pública. 2003; 19:S29-S36.

8. Brown DW, Brown DR, Heath GW, Balluz L, Giles WH, Ford ES, Mokdad AH. Associations between Physical Activity Dose and Health-Related Quality of Life. Med Sci Sports Exerc. . 2004; 36:890-6

9. Paffenbarger RS Jr, Lee IM. Physical activity and fitness for health and longevity. Res Q Exerc Sport. . 1996; 67: S11-28.

10. Shephard RJ. Habitual physical activity and quality of life. Quest. 1996; 48:354-65.

11. Rojas R, Schlicht W, Hautzinger M. Effects of Exercise Training on Quality of Life, Psychological Well-Being, Immune Status, and Cardiopulmonary Fitness in an HIV-1 Positive Population. Journal of Sport \& Exercise Psychology. 2003; 25:440-55

12. Tomas-Carus $P$, Häkkinen A, Gusi N, Leal A, Häkkinen K, Ortega-Alonso A. Aquatic Training and Detraining on Fitness and Quality of Life in Fibromyalgia. Med Sci Sports Exerc. . 2007; 39:1044-50.

13. Spirduso WW, Cronin DL. Exercise dose-response effects on quality of life and independent living in older adults. Med Sci Sports Exerc. . 2001; 33:S598-608.

14. Sutherland G, Andersen MB, Stoove MA. Can aerobic exercise training affect health-related quality of life for people with multiple sclerosis? Journal of Sport \& Exercise Psychology. 2001; 23:122-35.

15. ABEP - Associação Brasileira de Empresas de Pesquisa Critério de Classificação Econômica - Brasil 2008. http:// www.abep.org/codigosguias/Criterio_Brasil_2008.pdf. (acessado em 10/maio/2009).

16. Mader U, Martin BW, Schutz Y, Marti B. Validity of Four Short Physical Activity Questionnaires in Middle-Aged Persons [With appendixes]. Med Sci Sports Exerc. . 2006; 38:1255-66.

17. Matsudo SM, Matsudo VR, Araújo T, Andrade D, Andrade E, Oliveira $L$, et al. Nível de atividade física na população do Estado de São Paulo: análise de acordo com o gênero, idade e nível socioeconômico, distribuição geográfica e de conhecimento. Rev Bras Ciênc Mov. . 2002; 10:41-50.

18. Ramos LR. Fatores determinantes do envelhecimento saudável em idosos residentes em centro urbano: Projeto Epidoso, São Paulo. Cad Saúde Pública. 2003; 19:793-7.

19. Rossi VEC. Perfil das pessoas com diabetes mellitus tipo 2 cadastradas no programa de assistência ao diabético de Passos - MG [dissertação]. Ribeirão Preto (SP): Escola de Enfermagem de Ribeirão Preto, Universidade de São Paulo; 1999.

20. Tavares DMS. Condições de saúde de idosos diabéticos [tese]. Ribeirão Preto (SP): Escola de Enfermagem de Ribeirão Preto, Universidade de São Paulo; 2001.
21. Franco LJ. Epidemiologia do diabetes mellitus. In: Lessa I, organizador. O adulto brasileiro e as doenças da modernidade. São Paulo: Hucitec; Rio de Janeiro: ABRASCO; 1998. p. $123-38$.

22. Schaan BD, Harzheim E, Gus I. Perfil de risco cardíaco no diabetes melito e na glicemia de jejum alterada. Rev Saude Publica. 2004; 38:529-36.

23. Pelegrini A, Silva CR, Petroski EL, Benedetti TRB. Diabetes mellitus auto-referido e sua associação com excesso de peso em idosos. Rev Bras Cineantropom Desempenho Hum. (Online). 2011; 13:442-7

24. Guimarães FP de M, Takayanagui AMM. Orientações recebidas do serviço de saúde por pacientes para o tratamento do portador de diabetes mellitus tipo 2. Rev Nutr. 2002; 15:3744.

25. Cotta RMM, Batista KCS, Sena Reis RS, Souza GA, Dias G Castro FAF et al. Perfil sociossanitário e estilo de vida de hipertensos e/ou diabéticos, usuários do Programa de Saúde da Família no município de Teixeiras, MG. Ciênc Saúde Coletiva. . 2009; 14(4):1251-60

26. Souza, LJ, Chalita FEB, Reis AFF, Teixeira CL, Gicovate Neto $C$, Bastos DA et al. Prevalência de diabetes mellitus e fatores de risco em Campos dos Goytacazes. Arq Bras Endocrinol Metab. 2003; 47:69-74.

27. Grillo MFF, Gorini MIPC. Caracterização de pessoas com Diabetes Mellitus Tipo 2. Rev Bras Enferm. 2007; 60:49-54

28. Tavares DMS, Rodrigues FR, Silva CGC, Miranzi SSC. Caracterização de idosos diabéticos atendidos na atenção secundária. Ciênc Saúde Coletiva. . 2007; 12(5):1341-52.

29. Chaimowicz F. A saúde dos idosos brasileiros às vésperas do século XXI: problemas, projeções e alternativas. Rev. Saúde Pública. 1998; 31(2):184-200.

30. Lima-Costa MF, Barreto S, Giatti L. A situação socioeconômica afeta igualmente a saúde de idosos e adultos mais jovens no Brasil? Um estudo utilizando dados da Pesquisa Nacional por Amostra de Domicílios - PNAD/98. Ciênc Saúde Coletiva. 2002; 7:813-24

31. Davidson MB. Tratamento do paciente diabético no consultório.In: Davidson MB. Diabete Mellitus: diagnóstico e tratamento. 4ª ed. Rio de Janeiro (RJ): Revinter; 2001.

32. Sociedade Brasileira de Diabete. Consenso brasileiro sobre Diabetes 2002: diagnóstico e classificação do diabetes melito e tratamento do diabetes melito tipo 2. Rio de Janeiro (RJ): Diagraphic; 2003.

33. Sociedade Brasileira de Hipertensão; Sociedade Brasileira de Cardiologia. Diretrizes brasileiras de hipertensão arterial. Campos do Jordão (SP): SBC; 2002.

34. Tuomiletho J. Prevention of type diabetes mellitus by changes in lifestyle among subjects with impaired glucose tolerance. New Engl J Méd. 2001; 344(8):1343-50.

35. Ministério da Saúde (BR). Departamento de Atenção Básica. Área Técnica de Diabetes e Hipertensão Arterial. Hipertensão Arterial Sistêmica (HAS) e Diabetes mellitus (DM): protocolo/Ministério da Saúde. Brasília (DF): Ministério da Saúde; 2001.

36. The United Kingdom Prospective Study. Effect of intensive bloodglucose control with metformin on complications in overweight patients with type 2 diabetes: UKPDS 34 . Lancet 1998; 352(12):854-65. 
37. Silva DK, Nahas MV. Atividade física habitual e qualidade de vida relacionada à saúde em mulheres com doença vascular periférica. Rev Bras Ciênc Mov. 2004; 12(4):63-8.

38. Adams K, O'Shea P, O'Shea KL. Aging: its effects on strength, power, flexibility, and bone density. Natl. Strength Cond. Assoc. J. 1999; 21:65-77.

39. Brill P, Macera C, Davis D, Blair S, Gordon N. Muscular strength and physical function. Med. Sci. Sports Exerc. 2000; 32:4126.

40. Fiatarone M, O'Neill E, Doyle N, Clements K, Roberts S, Kehayias $\mathrm{J}$, et al. Exercise training and supplementation for physical frailty in very elderly people. N Engl J Med.1994; 330:1769-75.

41. WORLD HEALTH ORGANIZATION. Physical activity. Disponível em: <www.who.int/dietphysicalactivity/media/en/gsfs_ pa.pdf>. Acesso em: 05 jan. 2010.
42. Hallal PC, Victora CG, Wells JC, Lima RC. Physical inactivity: prevalence and associated variables in Brazilian adults. Med. Sci. Sports Exerc. 2003; 35:1894-900.

43. Caspersen CJ, Kriska AM, Dearwater SR. Physical activity epidemiology as applied to elderly populations. Baillieres Clin Rheumatol. 1994; 8:7-27.

44. Brach JS, Simonsick EM, Kritchevsky S, Yaffe K, Newman AB. The Association between physical function and lifestyle activity and exercise in the health, aging and body composition study. J Am Geriatr Soc. 2004; 52:502-9.

45. Young DR, Masaki KH, Curb JD. Associations of physical activity with performance- based and self-reported physical functioning in older men: the Honolulu Heart Program. J Am Geriatr Soc.1995; 43:845-54. 